\title{
ARCHIVO BIBLIOGRAPHICO \\ DA \\ FaCULDADE DE DIREITO DE S. PALLO \\ 1909
}





\section{CATALOGO DAS PIBLLCAÇ̄OES RECEBIIAS NA BIBLLOTHECA}

\section{Adquiridas por compra}

68x4 Albuquerque Sobrinho (Diogo Velho C.

D814 AlBUQUER Regimem Hypothecario. Porto Alegre I908. Broch. I

Vox.

6789 Almanack Garnier, para o anno de 1909. Cartonado.

4990 Baudry Lacantrénerie. Des Personnes vol. $3 .^{\circ}$ e $4 .^{\circ}$ Paris 1902 e 1905. Encad. 2

4990 》Des Obligations, vol. I. ${ }^{\circ}$ Paris 1907. Encad.

5239 Bonelli Commentario al Codice di ComMERCro. Firenza, não tendo data, continuando em publicação. Broch. 8 fasciculos

68I5 Branco. Diccionario Portuguez-Latino. $3{ }^{2}$ edição. Lisboa 1897. Encad.

6817 Consolidacăo das Leis Referentes á Justiça Federal. Rio de Janeiro i899. Broch.

68I9 Cornil. (G.) Traité de la Possession dans le Droit Romain. Paris 1905. Encad.

6730 DARESTE (R.) Nouvelles E'tudes d'istoire du Droit. Tome $3 .^{\circ}$ Paris 1907. Encad. 
VoL.

6820 ERrena (PAul). Traité de Droit Public Belge. Paris I909. Encad.

682 I Esmern. Elements de Droit Constitutionnel $4 .^{\mathrm{a}}$ édition. Paris I906. Encad.

6823 Fabreguetites. (P). Traité des Délits Polztiques. Paris Igor. Encad.

5589 Filippi. Medrcina Legale. Milano, não tendo data, e continuando em publicação. Fasciculo 35 e 36 Broch. 2 fasciculos.

I020 Gluck. Commentario alle Pandette. vol. 27, 29, 37 e 38, Milano, igo8 e igo9. Encad.

6825 GoOdnow. (Frank J.) Les Principes du Droit Administratif des Estats Unis. Paris I907. Encad.

6826 Grasserie. Des Principes Sociologiques de la Criminologie. Paris Igor. Encad.

6828 Ihering. Ouvres Choissies. Paris r893. Encad. 2

6829 Inglez de Souza. Direito Commercial. S. Paulo I893. Encad.

6830 João Cabral. Evolução do Direito Internacional. Rio de Janeiro Igo8. Broch.

683I Jouitor (L.) Simples Explications sur le Droit Civil et Bases Necessaires. Paris I904. Encad.

527 I Jurisprudencia do Tribunal Federal. Rio de Ja neiro IgOI. Broch.

6832 LACERDA. Diccionario Encyclopedico da Lingua Portugueza. 5.“ edição. Lisboa I878-1879. Encad.

Ior9 Leis do Brazil. Rio de Janeiro I808, I809 á I8I 5, I8I9. I824, I825' e I907. Broch.

I022 Leis Provinciaes. S. Paulo ı 877, I878, I880 á I888. Broch.

6834 JoCARD (EDMUND). L'identification des Récidiviste. Paris I909. Encad. 
6835 Molinier (Wictos). Traité Thearique et

Pratique de Droit Pénel. Paris 1893 e I894. Encad.

Vol.

6836 Mommsen (Theodoro). Manuel Antiquités Romarn. Paris 1907. Encad. 3

5360 Mortara. Commentario del Codice de Procedure Civil. Fasciculos 72, 73, 77 á 93. En proceguimento a publicação. I 8 fasciculos

68I3 Paul Bourget. Essais de Psychologie Contemporaine, não tendo a data da publicação. Paris. Broch.

6839 Rodrigo Octavio. Direito do Estrangciro no Brazil. Rio de Janeiro 1909. Hincad.

6840 Saleilles. Mélanges de Droit Comparée. Paris I907. Encad. .

684 I Sá Vianna. Elemento de Direito Internacional, vol. 1. ${ }^{\circ}$ Rio de Janeiro 1908. Broch.

5 I69 Scraloja. Dizionario Pratico del Deritto Privatto, não tendo a data, em proseguimento á publicação, n. ${ }^{\circ}{ }^{2}$ á 56.4 fasc.

6843 Vanni (IcIl,Io). Saggr di Filosofia Sociale et Giuridica. Bologna igo6. Encad.

6842 Vannr. Sezioni di Filosofia Del Diritto. Bologna 1908. Encad.

\section{Revistas e Jornaes}

4564 Diario Official da União. Rio de Janeiro I909. Broch.

II67. Direito (O). Revista Mensal de Legislação, Doutrina e Jurisprudencia. Rio de Janeiro I909. Broch. 3 fasciculos.

6803 Economista Brazileiro (O). Revista Semanal

de Economia, Financas, Politica e Litteratura. Rio de Janeiro I909. Broch. 2

4735 Gazeta de Noticias. Rio de Janeiro r9og. Broch. 4 
VoL.

4733 Jornal do Commercio. Rio de Janeiro I9o9. Broch.

2237 Journal des Economistes. Revue mensuelle de la Science économique et de la Statistique. Paris 1908. Broch.

5910 La Illustracion Sud Americana. Buenos Aires I909. Broch.

4264 L'Economiste français. Journal Hebdomadaire. Paris I9o9. Broch.

5909 L'Illustration. Journal Universel Hebdomadaire. Paris I909. Broch.

6683 Revista de Direito, por Dr. AnTonio Bento DE Faria. Rio de Janeiro I9o8, vols. 7, 8, 9, ro, e I909 vols. nrs. I I á I3. Encad.

4488 Revue Critique de Legislation ct Jurisprudence. Bruxelles rgog. Broch.

5426 Revue de Droit International et de Législation Comparée. Bruxelles igog. Broch.

5708 Revue D'Economie Politique. Paris 1909. Broch. I

4453 Revue des Deux Mondes. Paris :909. Broch. 6

\section{Adquiridos por doą̧ão}

2492 Affonso Celso (DR.) Resumo das Prelecões de Economia Politica. Rio de Janeiro I909. Broch.

6 igo Affonso Penna (Dr). Mensagem. Rio de Janeiro I909. Broch.

6igi Aguiar (General F. M. de Souza). Notas Biographicas. Rio de Janeiro I9o9. Broch.

6277 Americo Brasiliense Filho (Dr.) Memoria Historica da Escola de Pharmacia. S. Paulo I907-I908. Broch.

2008 Annaes da Camara dos Deputados. Rio de Janeiro I9o6. Broch. 
2006 Annaes da Camara dos Deputxdos do Estado de S. Paulo i9o9. Broch. 2

20I I Annaes do Senado do Estado de S. Paulo rgo8. Encad

VoL.

5022 Annuario da Escola Polytechnica de S. Paulo igo8. Broch.

6I92 Annuario da Faculdade de Philosophia e Letras. S. Paulo Igod. Broch

4II5 Annuario da Universidade de Coimbra I908-I909 Broch.

3770 Annuario do Ensino Publico do Estado de São Paulo I907-I908. Broch. 3 exemplares 3

6777 Annuario Estatistico de Uruguay i908. Broch. I

6or 5 Archino Bibliographico da Bibliotheca de Coimbra rgog. Broch.

6 I95 Asylo e Hospital Ophtalmico. S. Paulo I9o9. Broch

5975 Aureliano Coutinho (Desembargador). Discurso Inaugural do Curso Historico do Direito. S. Paulo I896. Broch.

6044 Boletim Telegraphico. Rio de Janeiro I9o9. Broch.

5973 Brasilio Machado (DR.). A Basilica da Apparecida. S. Paulo I909. Broch. . I

5972 Brasilio Machado (DR.). O Enxoval de Jesus. Broch.

68r6 BRuno. A Dictadura. Rio de Janeiro igog. Broch.

5977 Carvalho Fillho (Dr. José E. Freire de). Noticia Historica da Faculdade de Medicina da Bahia. I9o9. Broch.

679I Centro Industrial do Brazil, suas Riquezas Naturaes. Rio de Janeiro $190933^{\circ}$ vol. Broch. 
Vor.

6807 Congresso Scientifico Latino Americano. (Relatorio). Rio de Janeiro I909. Broch. 2

6818 Constituição Politica do Estado de S: Paulo. I908. Broch.

5983 CRUZ (J. Francisco DA) Ensaio Methodologia Didactica. Jahú I908. Broch.

6227 Descobrimento (O) das Aguas Mineraes da Pedreira Itaimbe. I908. Broch. .

6792. Divisão Administrativa e Municipal do Estado de S. Paulo igo8. Broch.

624I Domingos Barros. Aspectos. Rio de Janeiro I908, Broch.

I285 Estatutos da Companhia de Seguros de Vida Americana. Rio de Janeiro I9o8. Broch.

I285 Estatutos do Instituto de Protecçâo e Assistencia a Infancia. Rio de Janeiro I9o8. Broch. I

2823 FElippo Virgili. Manual de Estatistica. Rio de Janeiro I908. Cartonado.

I294 FERreira PINTO. Contra o Divorczo. Rio de Janeiro. I909. Broch.

598I Freire da Silva. (BACHAREl Augusto) Systema de Tachygraphia. S. Paulo I903. Broch.

6824 Funding-Loan. Accordo do Brazil com os Credores Externos. S. Paulo I909. Broch.

5982. Henrique Lobo. Os Casamentos Abencoados. S. Paulo rgog. Brocli.

6827 Herméto Lima. A Identidade do Homem. Rio de Janeiro igo8. Broch. . I

3549 Itajahy (Dr. M. Baptista) Mensagem. Sergipe I909. Broch.

5985 Jaguaribe (Dr. Domingos). Instituto Psycologico. S. Paulo igo8. Broch.

3987 LAmy. De Tabernaculo, não tendo a data da publicação, falta o frontespicio da obra. Raro. Encad. 
1022 Leis do Estado de S. Paulo rgo6 e 1907. Broch. $\quad 2$ 4363 Leme (Pedro F. de A. Paes) Livro da Familia. S. Paulo 1909. Broch.

4I27 Lista dos Estudantes Matriculados nas aulas da Faculdade de Direito de Minas Geraes. r9o9. Broch.

4r27 Lista da Faculdade de Direito de Porto Alegre. rgog. Broch.

- Mappa da Republica dos Estados Unidos do Brasil. I908. Na sala da consulta, em um quadro.

6837 Pedro Lessa (Dr.) Dissertação e Polemica. Rio de Janeiro 1909. Broch.

6844 Philipps (E.) Direito Internacional Publico. Rio de Janeiro I909. Broch.

5r32 Pierre Dinis. Le Bresil au $X X^{e}$ Siécle. Paris I909. Broch.

6278 Praga de Gafanhotos. Rio de Janeiro Igo8. Broch.

6238 Regulamento para o Alistamento Militar. Rio de Janeiro I908. Broch.

2 I80 Relatorio da Camara Muncipal de S. Paulo I908. Broch.

2346 Relatorio da Estrada de Ferro Central do Brazil. Rio de Janeiro 1907. Broch.

2127 Relatorio da Secretaria da Agricultura do Estado de S. Paulo 1908. Broch.

2346 Relatorio das Aguas e Esgottos da Cidade da Franca I909. Broch.

6279 Relatorio de Inquerito sobre Crise da Lavoura. Recife 1909. Broch.

2176 Relatorio do Ministerio da Justiça e Negocios Interiores. Rio de Janeiro I909. Broch. I

2289 Relatorio dos Telegraphos dos Estados Unidos do Brazil. Rio de Janeiro I909. Broch. 
VoL.

2 I82 Relatorio e Synopse dos Trabalhos do Senado do Estado de S. Paulo 1908. Broch.

6807 Reunião do Congresso Scientifico Latino Americano (Relatorio). Rio de Janeiro I908. Broch.

5974 Teixeira (Capitão Luiz Candido). Manual do Processo de Injurias Verbaes. Porto Alegre 1891. Broch.

6767 Terceira Conferencia Internacional Americana, Actas e Documentos. Rio de Janeiro 1907. Broch.

6292 Tomada (A) de Contas aos Concessionarios de Obras de Porto e a Companhia de Docas cin Santos. Rio de Janeiro Igrg. Broch.

63I4 Veiga Filho (Dr. João Pedro da) Lições Inaugural e Theses geracs de Philosophia do Dircito. S. Paulo igo8. Broch.

38 Vetus Testamentum Gracce-Leipzig. I 887. Encad.

\section{Revistas}

$553^{8}$ Anales de la Universidade do Chile 1909. Broch. I

5537 Anales de la Universidad de la Republica Oriental del Uruguay rgor. Brocl..

6847 Archives Diplomatiques. Paris 1908. Broch. 7 fasciculos.

6422 Ave Maria. S. Paulo i909. Broch.

6203 Bolctim da Propriedade Industrial. Rio de Janeiro 1909. Broch.

6207 Boletim do Museu Commercial. Rio de Janeiro I909. Broch.

2305 Boletim Official do Estado de Sergipe, r884, 1903 e 1907. Broch.

62 ro Boletim Policial. Rio de Janeiro 1908 e rgog. Broch.'.' 
6251. Deutsch Zeitung. S. Paulo 1909. Broch.

VoL.

6822 Evoluçăo. Recife I908. Broch.

I

6248 Fazendeiro (O). S. Paulo 1909. Broch. I

6244 Gazeta Clinica. S. Paulo I909. Broch. I

6220 Gazeta Juridica. S. Paulo igo9. Broch. 3

6020 Imprensa Medica. S. Paulo I909. Broch. I

6425 Lavoura (A). Rio de Janeiro 1908. Broch. I

4477 Revista do Instituto Historico e Geographico Brasileiro. Rio de Janeiro Igog. Broch. 2

542I Revista da Faculdade de Direito do Recife I906 e 1907. Broch.

54 I9 Revista da Faculdade de Direito de S. Paulo I909. Broch. 3 exemplares.

5447 Revista da Faculdade de Direito do Rio de Janeiro I909. Broch.

629I Revista da Universidade de Tegucigalpa Igog. Broch.

5978 Revista Didactica. Rio de Janeiro I909. Broch. I

5979 Revista do Brasil. Bahia Igo6. Broch. I

6249 Revista do Club de Engenharia. Rio de Janeiro I909. Broch.

6430 Revista do Centro de Sciencias, Letras de Campinas 1909. Broch.

67 I8 Revista Forensc. Bello Horizonte 1909. Broch. I

4487 Revista Maritima Brazileira. Rio de Janeiro Igng. Broch.

$65 \mathrm{I} 4$ Revista Medica. S. Paulo 1909. Broch. 2

6255 Revista Pharmacentica. S. Paulo I909. Broch. I

6545 Revista Polytechnica. S. Paulo r 909. Broch. I

3570 Santa Cruz. Revista Mensal, illustrada de Religiāo, Letras e Varicdades. S. Paulo I909. Broch.

I20I S. Paulo Judiciario. I909. Broch. 


\section{- $300-$ \\ Fornaes}

6338 Argos. S. Paulo i 909. Broch.

Vor.

6224 Bandeira Portuoueza Igo9 Broch. I

6359 Cachocirense. Cachoeira i909. Broch. I

4658 Cidade de Campinas 1909. Broch. 3

4730 Cidade de Santos rgog. Broch. 3

4I8I Commercio (O). de S. Paulo rgog. Broch. 4

4642 Constituinte (A). S. Paulo I879 e I909. Encad. 2

4642 Constituinte. Duplicata 2

4770 Correio Catholico. Uberaba rgog. Broch. I

4735 Correio da Manhã. Rio de Janeiro I909. Broch. 6

4532 Correio Paulistano. S. Paulo igog. Broch. 4

4532 Corrcio Pautistano Duplicata, Julho á Dezembro de I894. Encad. I

4558 Diario Mercantil. S. Paulo i888 i889 e 1890. Broch. 4

4693 Diurio Official do Estado de S. Paulo rgog. Broch.

4553 Diario Popular. S. Paulo 1909. Brocl. 3

4700 Jornal de Medicina. Pernambuco I909. Broch. I

6340 Jornal do Coinmercio. Juiz de Fóra 1909. Broch. I

4648 Minas Geraes 1909. Broch. 3

4725 Platéa (A). S. Paulo i909. Broch. 3

4600 Rebate (O). S. Paulo rgog. Broch. : I

625I Tempo (O). Campinas I9o9. Broch. 2

4729 Tribuna de Santos 1909. Broch. 3

6369 Tribuna da Franca 1909. Broch. I

S. Paulo, 3I de Dezembro de rgog.

O Amanuense,

Alfredo Dias do Rosario

\section{Confere,}

O Sub-bibliothecario,

Eugenio Manuel de Toledo. 